\title{
The family practitioner family: the use of metaphor in understanding changes in primary health care organizations
}

Tony Warne and Sheila Stark The Manchester Metropolitan University, Faculty of Community Studies, Law and Education, Department of Health Care Studies, Manchester, UK

\begin{abstract}
Current UK health policy guidance locates primary care at the frontiers of health care modernization. New organizational structures have resulted in general practitioner (GP) practices being brought together in Primary Care Groups (PCGs) and Primary Care Trusts (PCTs) each serving a much larger population group than the traditional GP practice. These changes have been accompanied with a need to explore new ways of working and thinking. This paper draws upon the experiences of nurses and GPs participating in an evaluation of workforce planning issues in primary health care. It explores how practitioners working in PCGs across one geographical area were able to gain a better understanding of what these changes, to both the structure and process of practice, might involve. During this developmental process the respondents used 'the family' metaphor, as a form of 'shorthand' to orientate themselves to the new responsibilities, challenges and opportunities presented by these changes to primary health care. It was in the use of terms and constructs that were familiar to their 'everyday life' experiences that made taking the first tentative steps in the change process easier. This paper suggests that using metaphors may be a powerful tool for policy makers, practitioners, managers and for researchers as they seek to communicate a plan for change and in understanding what these changes might mean.
\end{abstract}

Key words: change; families; metaphor; process; structure; understanding

\section{Introduction}

Much of the international health care community has been involved in a pandemic and sustained reform of its health care systems over the last two decades (Warne, 1999). In the UK, the National Health Service (NHS) has undergone continuous structural changes in its organization and orientation. These changes have reflected a paradigmatic shift in service focus and delivery away from the secondary care sector towards a renaissance of a primary and community care-led NHS (DoH, 1997). Current policy guidance locates primary care at the frontiers of health care modernization

Address for correspondence: Dr Tony Warne, The Manchester Metropolitan University, Faculty of Community Studies, Law and Education, Department of Health Care Studies, Elizabeth Gaskell Campus, Hathersage Road, Manchester M13 0JA, UK. Email:A.R.Warne@mmu.ac.uk
(DoH, 1997; 2000). New organizational structures have resulted in general practitioner (GP) practices being brought together first, as Primary Care Groups (PCGs), and subsequently as Primary Care Trusts (PCTs). Both these new organizational forms respond to a much larger population group than the more traditional GP practice. PCTs are set to become the driving force for change across the NHS, with much of the responsibility for commissioning and providing health care shifting to PCTs. These changes to primary health care organizations have been accompanied with a need to explore new ways of working and thinking (DoH, 1997; 2000), by establishing a shared understanding of the national and local strategic policy objectives (Warne, 1999).

Given this turbulent health care context (Stark et al., 2000), that currently appears to be relentless, developing beneficial ways for individuals to better understand these changes in order to move the 
government's agenda forward is necessary. This paper offers the potential of metaphors as 'enabling devices' as one such way. We argue that this can be part of an approach in allowing health care professionals to begin to 'see' their culture differently - to make the familiar strange (Hammersley and Atkinson, 1997), as well as making the strange familiar. In so doing, we argue such enabling devices can communicate and develop understandings of the nuance and complexity in everyday health care contexts (Lakoff and Johnson, 1995), as well as the exploration of new possibilities. Further, we illustrate this by providing an example of a metaphor (the family) that was used initially as a form of conceptual shorthand, additionally being brought to bear as an enabling device in the first step towards enabling practitioners, working in PCGs, to reconceptualize their practice context.

\section{Methodology}

The data included in this paper emanated from a larger evaluation of a workforce planning strategy (Warne etal., 1999). This was a study commissioned by the Merseyside Education and Training Consortium in 1998 and completed in 1999. In the course of collecting these data we found an unanticipated finding in the use of metaphor. To contextualize how these data were collected an overview of the methodology of the wider evaluation is presented here. The study was undertaken within three Health Authorities (HAs) located in the north west of England. The study centred specifically around workforce planning issues of role boundaries, professional development, attitudes towards and experiences of integrated teamworking, as these might be affected by the new and emerging organizational forms of national health and social care. Both quantitative and qualitative data were collected using a multimethod approach. This included 12 focus groups (107 participants), 18 semi-structured interviews (22 participants), and a survey sent to 210 respondents, with a response rate of $96(47 \%)$.

The sample for the survey was mainly practice nurses $(50 \%)$, but also included specialist community nurses (26\%), GPs (24\%) and other groups such as PCG Board members, HA managers and planners. Most participants $(69 \%)$ had 6 to 10 years of experience in their current positions, with some $14 \%$ being in their current positions over 20 years $(11 \%$ of these were GPs). Of participants $46 \%$ were employed by a Community Trust, $26 \%$ by a GP practice and the remainder by one of the three HA. The male participants $(22 \%)$ were nearly all GPs, but not all GPs participants were male). The samples for the focus groups and individual interviews were taken from the sample of survey respondents. Representation from each of the health care professionals listed above was included.

\section{Unanticipated finding}

The findings of the larger evaluation are published elsewhere (Warne et al., 1999). Here, we will explore an unanticipated finding (the use of metaphor) and its development and use as an approach to enable individuals to understand and respond to organizational change.

After completion of the evaluation we presented our findings to the stakeholders at various venues, including representatives from the various PCGs and potential PCTs (most of whom had been participants in the evaluation). These meetings were the first stage in an action learning approach to future organizational development (Revans, 1980). Our message was one that emphasized 'equality' and 'diversity' in both the development of new structures and processes for working, which would help ensure more productive and collaborative working. Originally, we advocated a 'wholesystems' approach to achieving these aims. This approach was derived from a synthesis of organic and mechanistic 'systems' concepts, often used in describing how organizations and the individuals within them interact with each other and other organizations (Trist, 1984; Wilson, 1999). For example, one of the recommendations reflected the need for the various respondents to work towards the establishment of new organizational identities as they came together first as PCGs, and eventually, as they became PCTs. These recommendations emphasized addressing the relationship transformations (the felt experience of the movement between dependence, independence and interdependence (Warne, 1999)) created by the social actions of individuals working across networks of professional groups in times of organizational change. However, after a couple of presentations 
we felt the message was not being received. Both formal and informal discussions during and after the sessions revealed that the audience did not understand the structures, or the processes, in relation to the current and future work cultures of PCG/Ts. As a result, we went back to our data and decided to incorporate into our presentations the language they were using when describing their work environment and relationships.

Using analytical memos (Wolcott, 1994) developed as part of the data analysis process, we noted that across all the professional groupings, the participants in their accounts, either explicitly or implicitly, used the concept of a 'family' to illustrate a point they were making. When discussing their current work culture, for example, many of the respondents (44\%), described their experience of working within a GP practice as like being part of a traditional family ( $70 \%$ of these respondents were GPs). The example illustrates how this particular metaphor became a form of conceptual shorthand for accessing different contextual understandings of the current organizational structures and process that participants experienced in their practice. Revisiting the data and analysis in this way revealed the metaphor's use by the participants was largely based around an uncomplicated representation and definition of nuclear and extended family groups. Each was being used in an 'everyday' way, a ubiquitous element of the language used. It was these familiar notions of kinship networks, reliance on reciprocity and family solidarity, which we felt could provide the basis for increasing the level of shared understanding among the participants involved in working through the change agenda. It appeared possible to use the metaphor of the traditional family as an enabling device to reveal these entangling strings of reciprocity, obligation, solidarity and coevolution in a way that the previous 'whole systems' metaphor apparently failed to do.

Thus, in the remaining feedback sessions and subsequent development meetings with the stakeholders, we drew upon the metaphor in order to explore how, where, why and in what way the new PCGs would be able to work at achieving a 'familial solidarity' in the new PCT framework. For example, in the respondents' accounts of who took responsibility for leadership and what this represented classically in terms of authority and power within the PCG was contextualized by an exploration of similar behaviours and consequences within the respondents own families. This appeared to promote a greater recognition of the importance of developing a shared value system which could influence their activities, both in how they operated as a 'family' and in how they formed, maintained and developed relationships with others outside of this immediate group. We would argue this was an important first step as the structure and processes of primary health care services moves from individual organizations to multiorganizational entities in the shape of PCG/Ts.

Our emergent strategy, therefore, was to take the conceptual shorthand (represented by the family metaphor), and to deliberately employ this as an enabling device; thus providing a more effective way of getting the participants to understand concepts that were unfamiliar to them in a familiar way. We expand on this process in the latter part of this paper and illustrate it using data from the evaluation. Exploring the congruence between the unintentional and intentional use of metaphor provides the starting point for our discussion.

\section{Metaphor mechanisms}

Metaphors are creative of meaning (Harré, 1986). Their use involves a process of discovery or invention, and they can provide new ways of viewing and experiencing the world we live in. Following Lakoff and Johnson (1995) and Turner (1996) we adopt the term metaphor by way of its broadest definition. Thus, our use of the term metaphor includes related concepts of analogies but does not embrace concepts such as metonymy, which relies more completely on substitution. In our usage, metaphor is a form of language, used in order to further greater understanding. Morgan (1993: 601) has argued that metaphors are a "basic structural form of experience, through which human beings engage, organize and understand their world'. It can be asserted, therefore, that metaphors and analogies can help liberate the imagination, foreground alternative conceptions of reality by selectively highlighting certain features of it and, thus, guide social action accordingly. In so doing metaphors can provide helpful interpretive schemes to aid the reduction of equivocality and can help individuals and groups better deal with ambiguity (Morgan, 1993). 
The use of metaphor as an effective tool in both research and organizational development has a long history in organizational and sociological studies (Clark, 2000). Many health care practitioners continue to work in organizations typified by explicit boundaries and/or implicit norms that inhibit the understanding of the interrelationships of the different players (Payne, 2001). This is a long-standing problem and there are many texts rich in metaphoric language that describe both organization and the organization in mechanistic, ecological, mythological and economic terms (Burrell, 1997; Clark, 2000; Handy, 1995; Lakoff and Johnson, 1995; Morgan, 1986). The use of metaphors in such examples being justified on the basis of facilitating greater understanding of the complex relationship of a number of different factors that can influence the performance of the individual and/or the organization. However, it could be argued that in doing so, individuals have often relied too heavily on idealized sequences of organizational action without sufficient attention being paid to how such sequences of action are affected by organizations in action. This can be demonstrated by the recent use of the inclusive metaphor used in promoting a more high profile role for nurses in the modernization of the NHS. The Working Together (DoH, 1998) guidance, aimed at raising the professional profile of nurses and improving the terms and conditions of employment advocated, among other strategies, 'family friendly policies'. This involved the consideration of developing more flexible methods of employment for those employees who had children - different shift patterns; time off to care for sick children, etc. Implementation of this idea proved to be relatively divisive across the workforce as single workers, or those without children, felt disadvantaged as a group. Thus, the consequence was a rather more 'messy reality' than the idealized sequencing promoted by the metaphors ('working together' and 'family friendly') perhaps suggested. In health care organizations, such 'messiness' and dissonance in the idealized sequencing of action has often attracted metaphoric comment. For example, at the microlevel of direct clinical work, Beattie (1995) used the metaphor of tribes to describe the warring fractions of many multidisciplinary teams and McKee et al. (1997) who at the organizational, or mesolevel, employed the metaphors of puppets and puppets masters to describe doctors (particularly hospital consultants) involvement in management decision making in the post-1990 reforms. Further, at the macrolevel of governmental policy making, Light (1998) uses the metaphor of the Trojan Horse in describing the introduction of the quasi-market to the UK health care system. More recently, the Shifting the Balance of Power (DoH, 2001) White Paper provides yet a further example of metaphor usage aimed at making macro-policy objectives accessible to those working at the microlevel who will be responsible for bring about the proposed changes.

At the wider and more generalized level, metaphor has formed the diet of embodiment discourses (Iphofen, 2000), perceptions of illness and health (Sontag, 1989) and in the social policy ideologies of welfare development (Lavette and Pratt, 1997). A shared input-process-outcome approach to the use of metaphors in these examples is achieved through the provision of a form of conceptual shorthand which aids understanding, and which is based upon a wider and inclusive shared language. Thus, in the Trojan Horse example (input) an individual does not need to have a degree in social policy or economics (process) to understand the implied risk of privatization of the NHS being brought about surreptitiously (output). The intentional and unintentional application of metaphors as illustrated by these examples highlight both the challenges and opportunities involved in establishing the metaphor's significance in use. We argue, however, that the use of metaphors is not without risk. Metaphors are in themselves not facts, and they are not the phenomena. Thus, it is vital to remain sensitive to the danger inherent in their use. They are mediators of the world and if extrapolated too far can misrepresent, confuse and mislead instead of fostering the emergence of new understandings (Iphofen, 2000). Care also needs to be exercised in how and where metaphors are intentionally used, a concern foregrounded during the early reporting back meetings in our use of the whole systems metaphor.

\section{Metaphors in use - explicitly and implicitly}

As previously stated, both during the data collection, particularly the interviews and focus groups, and the feedback sessions, the use of 'the family' metaphor, as a conceptual shorthand, enabled many respondents to gain a better understanding of several workforce issues, such as: 
- Effective/ineffective patterns/flows of working;

- Relationship/s with and between colleagues;

- Use of power and influence;

- Hierarchical structures within their organization and, further, behaviour patterns that reinforce these hierarchies;

- Rituals and stereotypical behaviour;

- Language and discourses used.

Many of the respondents, for example, explicitly used examples from family life, such as bringing up children, setting up home, developing relationships with others (in-laws and/or grandparents), in making the connections between these issues and similar issues and problems experienced in everyday family life. In some accounts more implicit references were made relating to the family. For example, patriarchal domination was used to describe the male GP's attitude towards others in the practice (whether this was as a benevolent or remote figure) and it was seen to be a barrier to effective primary health care working. In some accounts, both explicit and implicit references to the family or the way in which families changed were made. For example, some participants in the newly formed PCGs expressed their reaction to what they described as, the 'shot-gun marriage' approach of being compelled to join a PCG in ways that appeared reminiscent of a nuclear family reinforcing their solidarity and independence when perceived to be under some kind of threat:

We have learnt to work together, we understand each other, we know whose in charge and where we are going, these new PCGs will destroy all this work, our practice is built upon trust, you can't get the same trust across lots of different practices just because the government says so.

(GP)

It was the apparent ease with which these metaphorical connections were being made (the conceptual shorthand) that inspired a different approach in how we started to present the outcomes of our analysis and broad areas for consideration by the various stakeholders. We took the participants metaphors and used these to illustrate what might be involved in managing their change agenda. Our deliberate use of metaphor in this way is not without precedent. Many examples, of the use of the 'family' as a metaphor in examining social action are to be found (Cox and Paley, 1997; Giddens,
1996; Stacey, 1990; Warne, 1999). It is a familiar [sic] concept; people do things, in, for, and because of families. Families have be likened to miniature societies in controlling and distributing resources, particularly those connected with health and caring (Iphofen and Poland, 1998). Such concepts were foregrounded in the way many participants described either their current relationships across the teams and/or those they were developing in the newly formed organizations. We were able to take a number of explicit metaphorical 'family' members that had been identified to illustrate the types of issues they needed to tackle. So grounding both our analysis of the issues and recommendations for future action in the family metaphor that provided the opportunities for greater understanding among the participants. For example:

The senior partner is definitely the father figure, I think our Practice Manager is like our Mother, you know if you really want to get something done, go and see her not Dr J, most of the time we get treated like kids, you know, be seen not heard, no only joking, its alright here.

\section{(Practice Nurse)}

Within the individual GP 'family' the senior partner was almost exclusively identified as a father figure (paradoxically, even where the senior partner was a female GP). This was a common perception, and clearly could have positive as well as negative interpretations. What was important at the feedback meetings was to 'reveal' these perceptions and to ask the participants to consider what the implications might be in a way that reduced the potential for offence being taken. Likewise, practice nurses were predominately seen as mother figures, and they appeared to do a lot of 'backroom' work, over and above what their role entailed; their wishes and needs were often seen to be subordinated to the GPs views. Again, using the metaphor we were able to start to get individuals to reflect on what they knew about different professionals roles in the team and what they needed to find out and why this knowledge might be important in developing the effective team. Interestingly, where there was an implicit metaphorical reference made to these parental roles, the role of the general practice nurse was strongly identified and linked to the individual GP practice in which they worked. 
We are like one big happy family here, we have grown up together, we trust each other, and even if we don't always get on with each other all of the time, it always easy to resolve things.

\section{(Specialist Practitioner)}

I think the better Practice Nurses have done well because there is a lot of confusion in the community nursing teams about what they can and can't do... we work things like that out with our GPs.

(Practice Nurse)

Our survey data (Warne et al., 1999) revealed only $19 \%$ of the GPs felt their practice nurse was an 'independent' practitioner, with only $31 \%$ of the nurses also agreeing to this. The 'closeness' of such relationships, again where both positive and negative interpretations could be made, provided the opportunity to raise the issues involved in becoming a PCG. For example, managers (including the few surviving practice and fund managers) were often viewed as 'Aunts and Uncles' - with important decisions about the life of the practice being taken by the senior partner with the other GPs. Given the potential of PCG/T future budgets, this was a perception that perhaps could be challenged. So we asked respondents to explore the lessons that could be learnt from thinking about how within families the decision-making dynamic can be shifted by children striving for independence or changes in family membership resulting from marriage, divorce or death, etc. Thus what possibilities might there be to both protect the positive 'experiences' of working at an individual GP practice whilst recognizing that new and more inclusive decision-making processes would have to be developed. This was not always easy, however, with some respondents working within PCGs adopting defensive positions, for example:

Integrated team working is desirable, but not at the expense of knowledge or expertise within existing roles.

\section{(Practice Nurse)}

Additionally, many staff working in the secondary care and community trusts were often given 'inlaw' status by PCG staff, with inferences made that they were not part of the insider family group. However effective such community and secondary staff were as practitioners they were not part of the nuclear family by right.

Practice Nurses work inside the practice and us District Nurses work out in the community, we both have to much work to think about doing anybody's else's.

(District Nurse)

We can challenge the GPs more than the Practice Nurses as we are protected by the [Community] Trust, even though I would still see myself as being part of the practice team.

(Health Visitor)

There were some exceptions to this perception. Participants working in the HAs adopted a more inclusive view of what the emerging PCG family should look like, albeit this was somewhat proprietarily flavoured:

Now we have PCGs its going to be important that all of our nurses out there have to come together as one nursing family to a practice population.

(Nurse Advisor)

This use of the family metaphor was particularly useful as collectively, HAs were seen as being like grandparents, with obligations and responsibilities being given and afforded accordingly.

The Health Authority are like your grandparents, they interfere in things they don't know about, make demands upon your time when you could be doing other thing. But we love them and when everything looks bad we can always go and talk to them.

(Practice Nurse)

In a family you are quite protected really, you can fall out with others, we can agree to disagree and that's fine... families can handle conflict well... we are stronger if we stick together and present a united front to say them from Primrose Hill [The Health Authority $H Q]$.

(Practice Nurse)

In capturing and using these metaphorically based perceptions we were able to get the respondents to consider what was likely to be involved in managing the changing HAs and PCG/PCTs relationships. Being able to get both organizations to 
consider these changing relationships also helped keep discussions focused on the patient. In all the discussion about changing structures and roles, sometimes the patient was lost to sight. Indeed, patients appeared to be given the role of children (seldom seen or heard in the practitioners' conversations as explicit members of the GP family) although as within many families, they were at least, rhetorically, given a much higher and important status.

Within the focus groups and interviews some of these metaphorical accounts also implicitly touched upon the extended nature of relationships with those outside of the family or where 'blood' appeared to be 'thicker than water'. Thus there was an implied system of relationships that reflected wider kinship contexts:

There still exists, almost, a culture of who you socialize with is who you refer to ... We have a consultant with serious attitude problem, everyone is aware of it, he's rude to patients and we got a number of complaints. ENT [Ear, Nose and Throat] services is available elsewhere and its cheaper, but he's very well established and coming up to retirement so our doctors keep referring to him.

(Practice Manager 2)

Dermatology is a hard one to change, anyway its been hopeless for years, it been appalling and you get all the jokes about the medical Mafia not wanting to change that much even if we wanted to try!

\section{(Practice Manager 4)}

Conceptualizing such relationships in this wider context also has a salience for the wider NHS modernization programme. It has been argued, that the GP practice has long been established as the 'centre' or 'gatekeeper' of the provision of health care (Gouldner, 1954), a position reinforced in current governmental modernization policies $(\mathrm{DoH}$, 1997; 2001). In this context, we argue that individually and collectively, GP practices are 'significant' social organizations in their own right. As these organizations merged together into multipractice PCGs and more recently towards the autonomous PCT status, a new identity is encouraged, based upon reciprocal and collaborative networks of multiprofessional, multiagency teams (Light, 1998; Payne, 2001; Warne, 1999). Thus, structurally and functionally PCG/Ts can be described as self-contained social organizations, metaphorically a family but who also form part of a wider community of practitioners (an extended family). These new 'families' are located functionally within the wider social systems of society. However, such systems are increasingly becoming more complex, demanding different forms of relationships between and across these systems.

\section{Metaphors matter}

In the context of health care, the changing emphasis in the relationships of those involved in these new approaches demands even greater effort and understanding from all those involved if some form of health gain is to be achieved. Realizing this objective is likely to be inhibited by a range of different world views, value systems, leadership prescriptions and unhelpful but powerful tribal boundaries (Stark et al., 2000). In such a turbulent environment, the use of metaphor has many attractions as an enabling device. As in the experience of our participants, for example, it helped them to begin to develop a 'map' upon which individuals and groups can locate the effects of previous, current and future actions. It is in this process of transition, where metaphor is used unintentionally as a form of shorthand to where the metaphor is intentionally used as an enabling device, that we argue metaphor has a great deal to offer.

In this paper we have argued that it was the family metaphor, used as a form of conceptual shorthand, that better enabled individuals to make sense of their experiences. However, in order to become an enabling device the family metaphor needed to be grounded in notions of the family ideal, that is the way in which individuals recognize family membership facilitates the individual construction of a reality which may yet to be realized. In a psychological and sociological context, these processes are the 'everyday' processes of socialization. Individuals interpret and order their experiences through various socialization and countervailing processes (Warne, 1999). It is often only within the boundaries of the intimate family relationships that these perceptions are given some validation. Caution, however, needs to be exercised here in extrapolating the family metaphor in this way. Laing (1966), whose work explored the 
relationships between family members and not those between the family and other social institutions, provides a useful check to the use of the family metaphor in the context of organizational dynamics. Laing saw alliances being formed and re-formed within individual families; family members playing each other off against others in achieving their own ends, and the opportunities for confusion, misunderstanding, deception, manipulation and attempted manipulation being manifold. There was some evidence of this occurring with our participants, particularly between the various nursing groups. Community nurses, for example, were viewed as being 'outsiders' by the practice nurses, with the practice nurses using this as a way of maintaining their relationship with the GPs. In contrast to Parsons (1951), who highlighted the needs of the social system, Laing emphasized the importance of autonomy, freedom and selfawareness, but also saw the family as being possibly suffocating and through the imposition of unremitting reciprocal obligations, ultimately restricting in the development of individuality. As individuals working in GP practices start to work at the changes resulting from the development of PCG/PCTs such tensions will need to be addressed. For example, how will the different practitioners both promote practice diversity whilst achieving the implementation of policy homogeneity?

These cautionary notes not withstanding, in the example used in this paper, the metaphoric use of the family was found to be useful for the stakeholders involved. Just as this metaphor was used to illustrate and enable understanding, its ephemeral nature implies that in other situations the use of metaphors might be as useful. For example:

- policy makers in getting the new message across

- practitioners in making sense of the new agendas for practice

- managers in facilitating the change process

- for researchers interested in understanding the basis of these various social actions.

Both as forms of conceptual shorthand and as enabling devices, metaphors offer one more way for individuals to gain a better understanding of their role, its relationship to others, the change agenda and the action needed to achieve such changes.

\section{Summary}

The NHS modernization agenda sets out an ambitious range of changes to the structure and functioning of the health care in the UK. Primary health care services are seen as the vanguard in leading many of these changes. Individuals working in primary care organizations have had to both develop an understanding of what is involved and work through an incremental process aimed at implementing these changes. This paper has explored how the use of metaphors can be used as enabling devices in these development processes. The use of a metaphor (the family) which was originally used as a form of conceptual shorthand by participants involved in working through these changes was explored. Although it was recognized that a definition of 'the family' remained illusive, it was argued that elements of the 'traditional' family were, metaphorically, useful on at least two levels: i) a conceptual level (as a form of shorthand). Allowing for a greater understanding of the various relationship transformations that are required as new organizational and professional structures are created and recreated. And ii) at a process level, as an enabling device for individuals and groups to start to explore new directions that both allow for solidarity and equality in recognizing diversity. Whilst this individual case study can only serve as an illustration of the potential of using metaphor in this way, given the enormous scale of changes facing health care practitioners, it maybe one more useful 'tool' in understanding and achieving the change agenda.

\section{Acknowledgements}

The authors thank the Merseyside Education and Training Consortium and participants from St Helens and Knowsley, Sefton and Liverpool Health Authorities for their contribution to this paper.

\section{References}

Beattie, A. 1995: War and peace among the health tribes. In Soothill, K., Mackay, L. and Webb, C., editors, Interprofessional relations in health care. London: Edward Arnold, 11-26. 
Burrell, G. 1997: Pandemonium: towards a retro-organization theory. London: Sage.

Clark, P. 2000: Organisations in action: competition between contexts. London: Routledge.

Cox, M. and Paley, B. 1997: Families as systems. Annual Review Psychology 48, 243-67.

Department of Health. 1997: The new NHS: modern and dependable. London: The Stationary Office.

Department of Health. 1998: Working together. London: The Stationary Office.

Department of Health. 2001: The NHS Plan. London: The Stationary Office.

Giddens, A. 1996: Modernity and self-identity. London: Polity.

Gouldner, A. 1954: Patterns of industrial bureaucracy. New York: Free Press.

Hackney, R., Dhillon, G. and McBride N. 1997: Primary care information technology within the NHS. International Journal of Public Sector Management 10, 388-95.

Hammersley, M. and Atkinson, P. 1997: Ethnography, second edition. London: Routledge.

Handy, C. 1995: Waiting for the mountain to move. London: Arrow.

Harré, R. 1986: Varieties of realism: a rationale for the natural sciences. Oxford: Blackwell.

Iphofen, R. 2000: Axioms and metaphors: sociological imaginings. BSA Network 35, 9-10.

Iphofen, R. and Poland, F. 1998: Sociology in practice for health care professionals. London: Macmillan.

Laing, R. 1966: The politics of the family and other essays. London: Tavistock.

Lakoff, G. and Johnson M. 1995: Metaphors we live by. Chicago: University of Chicago Press.

Lavalette, M. and Pratt, A. 1997: Social policy: a conceptual and theoretical introduction. London: Sage.

Light, D. 1998: Managed care in a new key: Britain's strategies for the 1990s. International Journal of Health Services 28, 427-44.
McKee, L., Marnoch, G. and Dinnie, N. 1997: Medical managers: puppetmasters or puppets? Sources of power and influence in clinical directorates. Conference Paper, York: British Medical Sociology Annual Conference.

Morgan, G. 1986: Images of organization. London: Sage.

Morgan, G. 1993: Imaginization: the art of creative management. California: Sage.

Parsons, T. 1951: The social system. London: Free Press.

Payne, M. 2001: Teamwork in multi-professional care. London: Macmillan.

Revans, R. 1980: Action learning: new technique for management. London: Blond and Briggs.

Rivett, J. 1998: From cradle to grave: fifty years of the NHS. London: Kings Fund.

Stacey, J. 1990: Brave new families. New York: Basic.

Stark, S., Stronach, I., Warne, T., Skidmore, D., Cotton, A. and Montgomery, M. 2000: Educational preparation for mental health nursing: outcomes evaluation of the contribution of mental health nurses within the multi-professional, multiagency team. London: English National Board for Nursing Midwifery and Health Visiting.

Sontag, S. 1989: Illness as metaphor and AIDs and its metaphors. New York: Picador.

Turner, V. 1996: Dramas, fields and metaphors. New York: Cornell.

Warne, T. 1999: Contracting and customs in the UK general practitioner fundholding scheme. Unpublished $\mathrm{PhD}$ thesis, Manchester Metropolitan University, Manchester.

Warne, T., Stark, S. and Street, C. 1999: Nursing in general practice: a study of workforce planning issues. Merseyside Education and Training Consortium, for the Sefton, St Helens and Knowsley and Liverpool Health Authorities, Merseyside.

Wilson, E. 1999: Consilience: the unity of knowledge. London: Abacus.

Wolcott, H.F. 1994: Transforming qualitative data: description, analysis and interpretation. California: Sage. 